\title{
INVESTIGATIONS INTO THE QUALITY OF IMAGE-BASED POINT CLOUDS FROM UAV IMAGERY
}

\author{
H.-J. Przybilla ${ }^{\text {a, }}$, M. Mindstaedt ${ }^{\text {b }}$, T. Kersten ${ }^{\text {b }}$

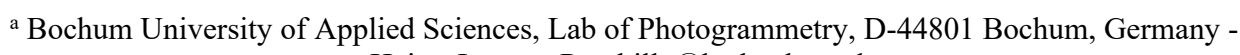 \\ Heinz-Juergen.Przybilla@hs-bochum.de \\ b HafenCity University Hamburg, Photogrammetry \& Laser Scanning Lab, Überseeallee 16, D-20457 Hamburg, Germany - \\ (Maren.Lindstaedt, Thomas.Kersten)@hcu-hamburg.de
}

\section{Commission ICGW I/II}

KEY WORDS: UAV aerial flight, test field, image data format, dense point cloud, data filtering, comparison

\begin{abstract}
:
The quality of image-based point clouds generated from images of UAV aerial flights is subject to various influencing factors. In addition to the performance of the sensor used (a digital camera), the image data format (e.g. TIF or JPG) is another important quality parameter. At the UAV test field at the former Zollern colliery (Dortmund, Germany), set up by Bochum University of Applied Sciences, a medium-format camera from Phase One (IXU 1000) was used to capture UAV image data in RAW format. This investigation aims at evaluating the influence of the image data format on point clouds generated by a Dense Image Matching process. Furthermore, the effects of different data filters, which are part of the evaluation programs, were considered. The processing was carried out with two software packages from Agisoft and Pix4D on the basis of both generated TIF or JPG data sets. The point clouds generated are the basis for the investigation presented in this contribution. Point cloud comparisons with reference data from terrestrial laser scanning were performed on selected test areas representing object-typical surfaces (with varying surface structures). In addition to these area-based comparisons, selected linear objects (profiles) were evaluated between the different data sets. Furthermore, height point deviations from the dense point clouds were determined using check points. Differences in the results generated through the two software packages used could be detected. The reasons for these differences are filtering settings used for the generation of dense point clouds. It can also be assumed that there are differences in the algorithms for point cloud generation which are implemented in the two software packages. The slightly compressed JPG image data used for the point cloud generation did not show any significant changes in the quality of the examined point clouds compared to the uncompressed TIF data sets.
\end{abstract}

\section{INTRODUCTION}

Various digital cameras are used for UAV aerial flights, ranging from the UAV provider's proprietary system to high-end cameras in medium format, such as those used in classic aerial photogrammetry. The image data is usually stored in lossy data compression format JPG. In addition to saving storage space, the main reasons for this are faster data storage during the aerial flight and the option of making corrections (e. g. radial symmetric distortion) directly on the image (Przybilla et al. 2017). In many cases the use of RAW image data is not considered because it cannot be processed directly, but an additional step of image conversion is necessary. TIF image data derived from RAW images store the image information loss-free and are also not geometrically pre-processed.

The aim of the present investigation is, on the one hand, to evaluate the influence of the image data format on point clouds generated within the framework of a Dense Image Matching process. The image data recorded at the UAV test field of the Zollern colliery (Fig. 1), set up by Bochum University of Applied Sciences, were taken with a medium format camera of the manufacturer Phase One (IXU 1000) (Phase One 2019a). The sensor has a resolution of $100 \mathrm{MPixel}$ and was used with a carrier platform from the company Coptersystems (2019) (Fig. 2). The system enables fast storage of RAW data from which both, uncompressed TIF and compressed JPG image data, were derived afterwards using the Capture One software (Phase One 2019b). For the following investigations, the JPG image data was compressed to $80 \%(100 \%=$ lossless $)$. On the other hand, the effects of different data filters on the point clouds, which are part of the two evaluation programs used (PhotoScan from Agisoft and Pix4Dmapper from Pix4D), are examined (chapter 2).

Questions about the quality of image-based point clouds were already discussed in various publications (Kersten \& Lindstaedt 2012, Kersten et al. 2016, d'Oleire-Oltmanns \& Lackner 2018, Zhang et al. 2018, Haala et al. 2019).

\section{GENERATION OF DENSE POINT CLOUDS}

The dense point clouds were processed with the software Agisoft PhotoScan (Agisoft 2019a) and Pix4Dmapper (Pix4D 2019a) on the basis of both generated TIF and JPG images. The quality of the camera used in this test, in comparison to proprietary systems of the UAV manufacturer DJI, is examined more closely on results of image triangulations, which is discussed in Przybilla (2019).

The computation of the dense point cloud is configured in the software by specifying a few parameters. To control the point density, primarily is information on the image scaling required, $i$. e. whether complete or resolution-reduced images are used in the calculation process. Further parameters aim at the reduction of outliers as well as the smoothing of the data. The point clouds used for these investigations are parametrized according to Table

\footnotetext{
* Corresponding author
} 


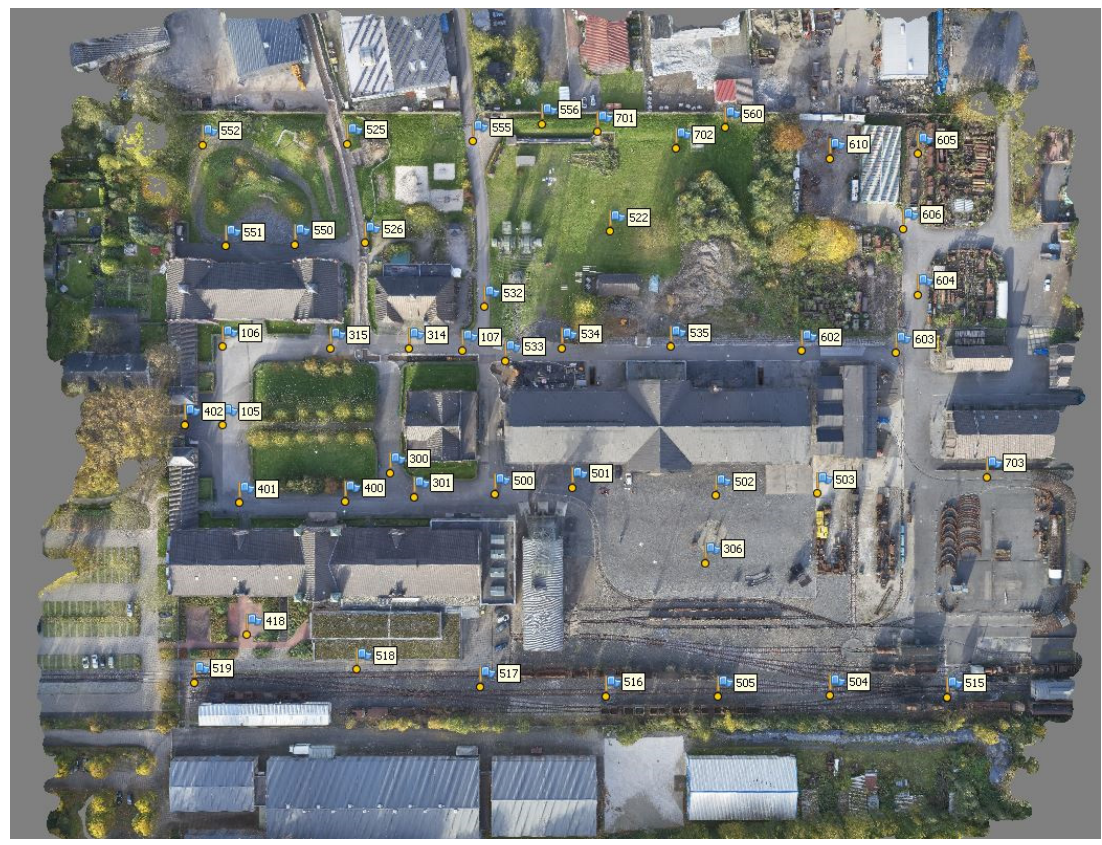

Figure 1. UAV test field at the Zollern colliery in Dortmund with control point distribution (used as check points $\mathrm{ChP}$ )
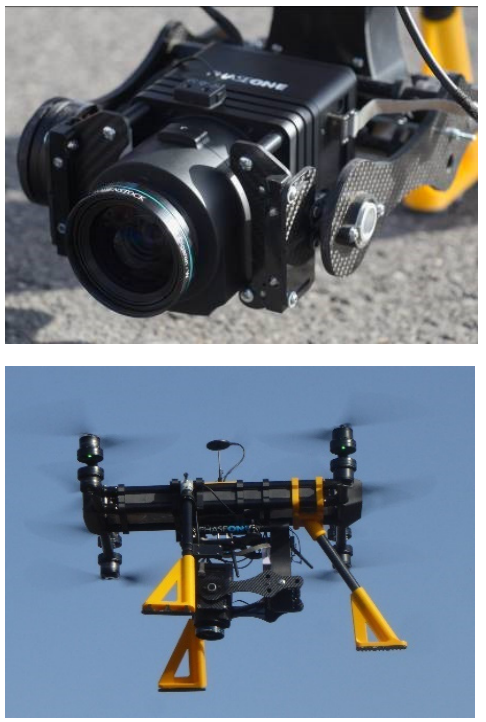

Figure 2. Phase One IXU 1000 (100Mpix) (top), Coptersystems Multicopter (bottom)

\begin{tabular}{|l|l|l|l|}
\hline Parameter & Setting & Description & Number of points \\
\hline Quality & Medium & Usage of each 4th pixel per row and column. & \\
\hline $\begin{array}{l}\text { Depth filtering } \\
\text { mode }\end{array}$ & Aggressive & $\begin{array}{l}\text { Small details are eliminated. Filtering out of most outliers (normal } \\
\text { setting for aerial image data). }\end{array}$ & 82.943 .385 \\
\hline & Moderate & Returns results that lie between the "mild" and "aggressive" option. & 86.708 .706 \\
\hline & Mild & $\begin{array}{l}\text { Small details are retained; important features are not detected as } \\
\text { outliers. }\end{array}$ & 82.797 .394 \\
\hline & Disabled & $\begin{array}{l}\text { This option is not recommended as the resulting dense point cloud } \\
\text { can be extremely noisy. }\end{array}$ & 83.918 .236 \\
\hline
\end{tabular}

Table 1: Parameterization of the dense point clouds in Agisoft PhotoScan - as described by the software developer (Agisoft 2019b)

\begin{tabular}{|c|c|c|c|}
\hline Parameter & Setting & Description & Number of points \\
\hline Image scaling & $\begin{array}{l}1 / 2 \quad \text { (half } \\
\text { image size, } \\
\text { default) }\end{array}$ & $\begin{array}{l}\text { Half size images are used to compute additional 3D points. It is the } \\
\text { recommended image scale. }\end{array}$ & \\
\hline \multirow[t]{4}{*}{ Point Density } & & This parameter defines the density of the densified point cloud. & \\
\hline & $\begin{array}{l}\text { Optimal } \\
\text { (default) }\end{array}$ & $\begin{array}{l}\text { A 3D point is calculated for each ( } 4 \text { /image scale) pixel. If image scale } \\
\text { is set to } 1 / 2 \text { (half image size), one } 3 \mathrm{D} \text { point is calculated every } 4 /(0.5) \\
=8 \text { pixels of the original image. }\end{array}$ & \\
\hline & $\begin{array}{l}\text { Option: } \\
\text { Multiscale } \\
\text { enabled }\end{array}$ & $\begin{array}{l}\text { Multiscale (default): If this option is enabled, additional 3D points are } \\
\text { calculated over multiple image scales, starting with the chosen scale } \\
\text { from the dropdown list "image size" up to the } 1 / 8 \text { scale ( } 1 / 8 \text { image } \\
\text { size, tolerant). }\end{array}$ & 92.282 .251 \\
\hline & $\begin{array}{l}\text { Option: } \\
\text { Multiscale } \\
\text { disabled }\end{array}$ & $\begin{array}{l}\text { In some cases, deactivating the Multiscale option produces less noise } \\
\text { in the point cloud. } \\
\text { This option should be disabled if there is a lot of noise visible in the } \\
\text { point cloud and artefacts are present in 3D texture mesh, DSM or } \\
\text { orthomosaic. }\end{array}$ & 70.574 .392 \\
\hline
\end{tabular}

Table 2: Parameterization of the dense point clouds in Pix4Dmapper - as described by the software developer (Pix4D 2019b)

1 and 2. Information about the parameterization of the dense point cloud generation is hardly given by the software developer. With regard to the adjustable image size (parameter: quality / image scaling), both providers pursue the same strategies by selecting appropriate reduction levels in addition to the full image format. This parameter has a significant influence on the size of the generated dense point clouds. Furthermore, the procedures differ in the fact that PhotoScan offers several variants of data filtering. This option is rather limited in Pix4Dmapper (only available by activating/deactivating the parameter "Multiscale"). 
If a lot of noise visible in the point cloud and artefacts are present, the option has to be deactivated according to the software provider's instructions.

To enable a comparison of the point clouds from both software packages, the quality parameters were selected in a way that point clouds of comparable sizes were obtained.

\section{TEST RESULTS}

The point clouds generated through the calculations form the basis of investigation. The existing Z-coordinates of the control points are used to determine height deviations from the dense point clouds. Moreover, linear comparisons of point clouds are carried out using several profiles. Finally, different point clouds are compared to references using selected surfaces that represent varying surface structures. In this comparison point clouds from terrestrial laser scanning are used as reference data.

\subsection{Pointwise comparisons}

For the pointwise comparisons, the shortest distance (in vertical direction) between the check points (ChP) and the dense point cloud is calculated. The distribution of the check points, which were used as control points in the aerial triangulation, is illustrated in Figure 1. As illustrated in Figure 3, the vertical deviations of the PhotoScan point cloud scatter slightly more than the corresponding deviations of the Pix4Dmapper point clouds. However, the average deviation is only $1 \mathrm{~mm}$, while the point cloud generated in Pix4Dmapper show a larger systematic negative deviation, which is on average about $8-10 \mathrm{~mm}$ above the check point height level. This value corresponds to approx. $2 / 3$ of the ground sampling distance (GSD) of $14 \mathrm{~mm}$ defined by the photo scale of the aerial flights. Significant deviations between point clouds based on different image data formats (TIF or JPG80) are not visible.

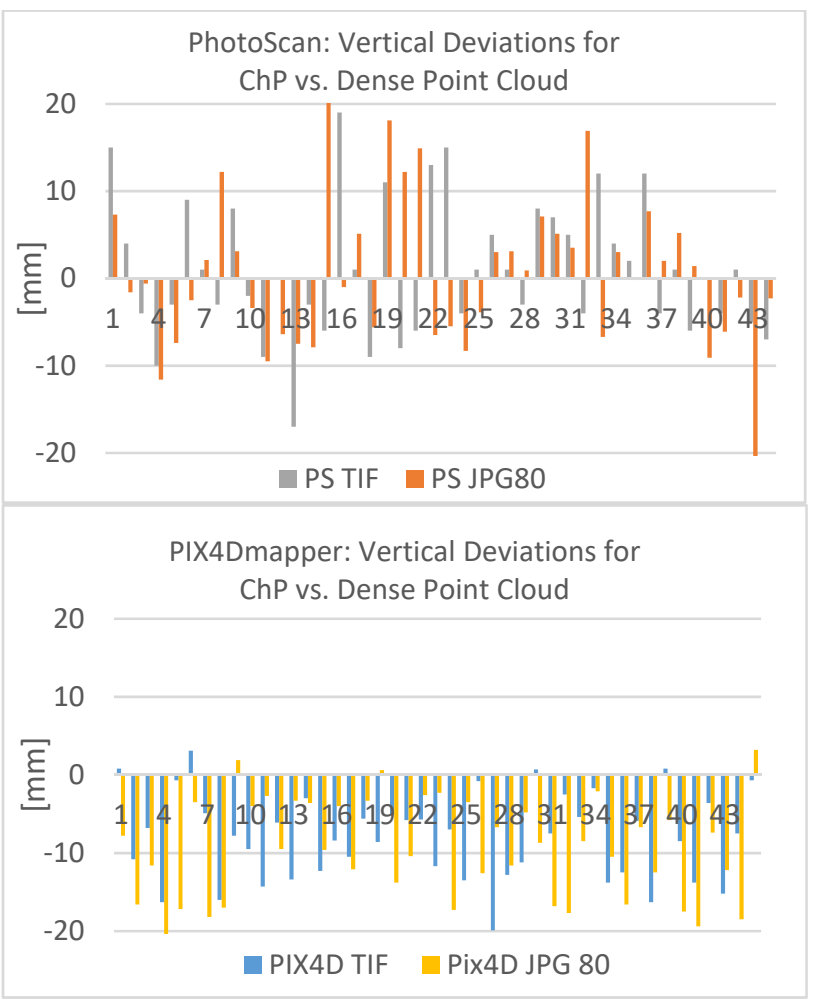

Figure 3. Vertical deviations between checks points and dense point cloud. Positive value - point cloud is below the $\mathrm{ChP}$; negative value - above the ChP. PhotoScan point cloud (top) and PIX4Dmapper point cloud (bottom)

\subsection{Profile-based comparisons}

Objects with characteristic features such as roof structures and rails were included in the linear comparisons (Figure 4). Figures 5-8 show the results of $3 \mathrm{D}$ point cloud comparisons of rail profiles in the longitudinal axis and transverse direction.

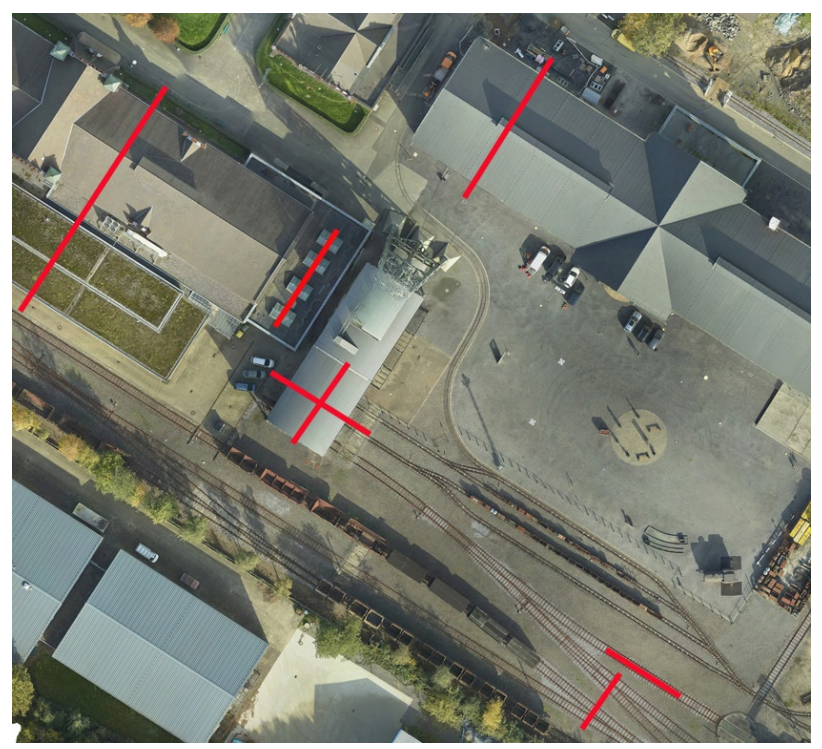

Figure 4. Distribution of profiles in the UAV test field

In Figure 5 the height offset of the profiles calculated with Pix4Dmapper is clearly recognizable in comparison to the profiles from PhotoScan. As already demonstrated in the pointwise comparisons, the Pix4D profiles are also systematically located above those of the PhotoScan computation. In Figure 6 the shape of each single profile is additionally depicted for better illustration. Fundamentally, the respective profiles of the two software packages correspond to each other, but the profiles of PhotoScan are obviously smoothed due to the applied filter factor of "aggressive".

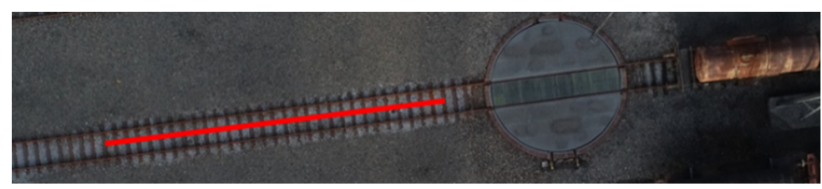

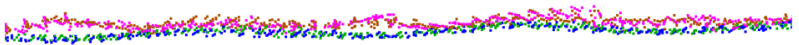

Figure 5. Presentation of the height profiles of the different point clouds in the track axis - profile position in the photo (top), point cloud profiles (bottom): P4D JPG80 (pink), P4D TIF (brown), PS JPG80 (green) and PS TIF (blue)

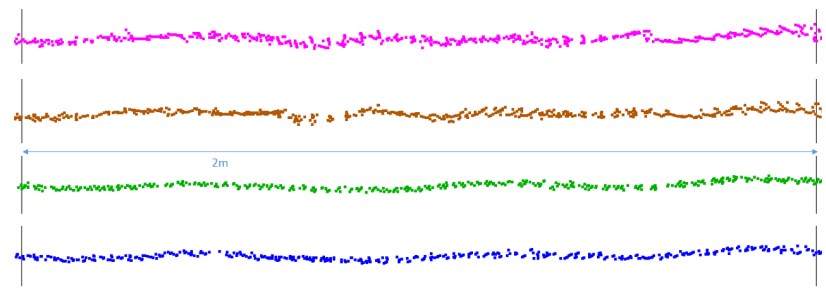

Figure 6. Individual representation of the height profiles from Figure 5 - P4D JPG80 (pink), P4D TIF (brown), PS JPG80 (green) and PS TIF (blue) 
The transverse profiles shown in Figures 7 and 8 confirm the previous statements. Again a clear smoothing of the PhotoScan profiles is visible. The profiles of Pix4Dmapper are basically more true to the shape of the rail tracks, but a higher scattering is apparent, which is probably caused by the activated parameter "Multiscale" (Table 2). Unfortunately the software developer's information on the effect of this parameter is not very concrete. However, the differences between the profiles generated using the different image data formats (TIF / JPG80) are negligible.

The present result raises the question of whether, using other filter settings in PhotoScan (Table 1), the rails can be imaged as clearly as in Pix4D. Therefore, a rail section with all four filter settings of PhotoScan was compared with the result of Pix4D. Surprisingly there were no significant differences between the profiles of the rail tracks and its surface structure using these different filter settings (Figure 9). The profile illustrations in Figure 10 also confirm the previous statements on height offset and filtering effects.

\subsection{Area-based comparisons}

The three test objects for the area-based 3D comparisons of the various point clouds are shown in Figure 11. The selected areas represent surfaces with varying surface structures. Point clouds scanned with the terrestrial laser scanning system Z+F IMAGER $5010 \mathrm{C}$ are available as reference data.

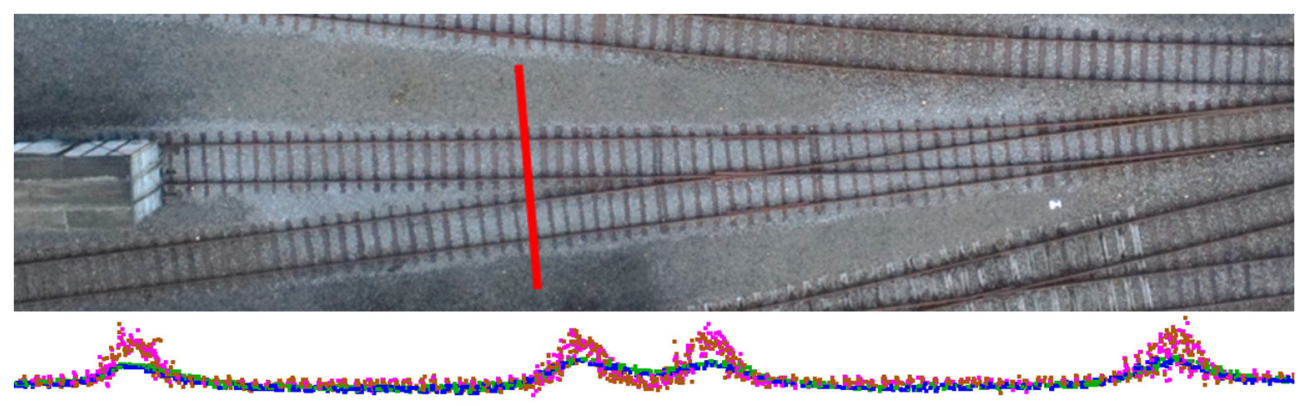

Figure 7. Profiles of the different point clouds perpendicular to the rail axis - profile position in the photo (top), point cloud profiles (bottom): P4D JPG80 (pink), P4D TIF (brown), PS JPG80 (green) and PS TIF (blue)

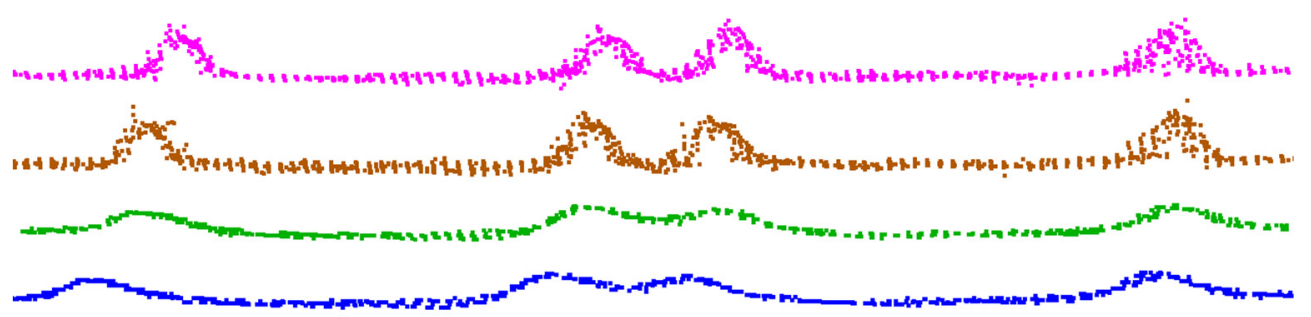

Figure 8. Presentation of each profile as shown in Figure 7:

P4D JPG80 (pink), P4D TIF (brown), PS JPG80 (green) and PS TIF (blue)

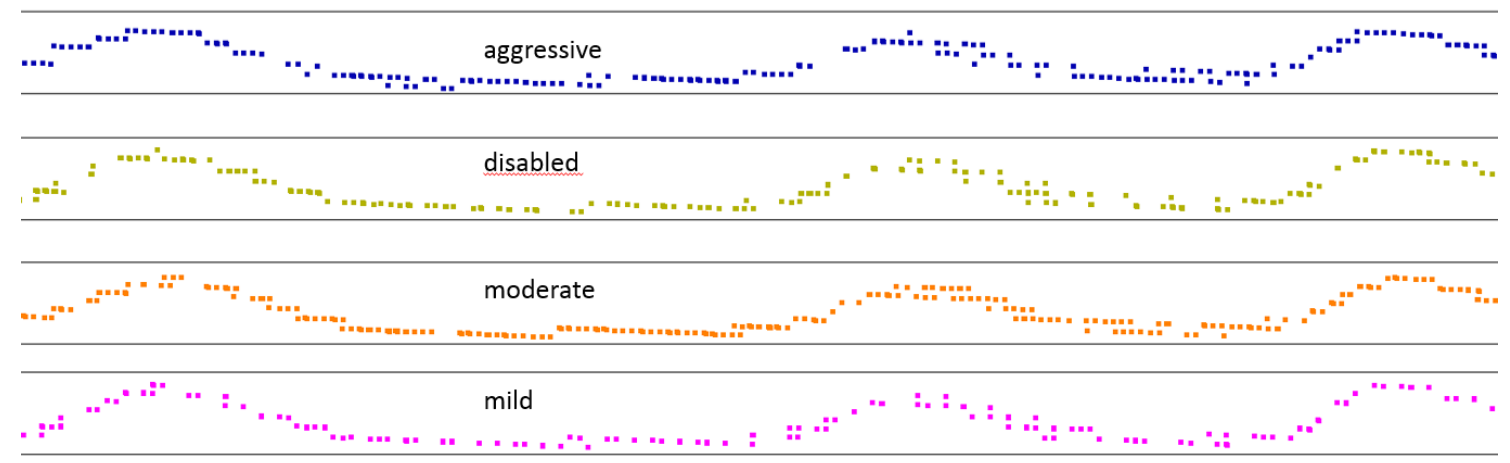

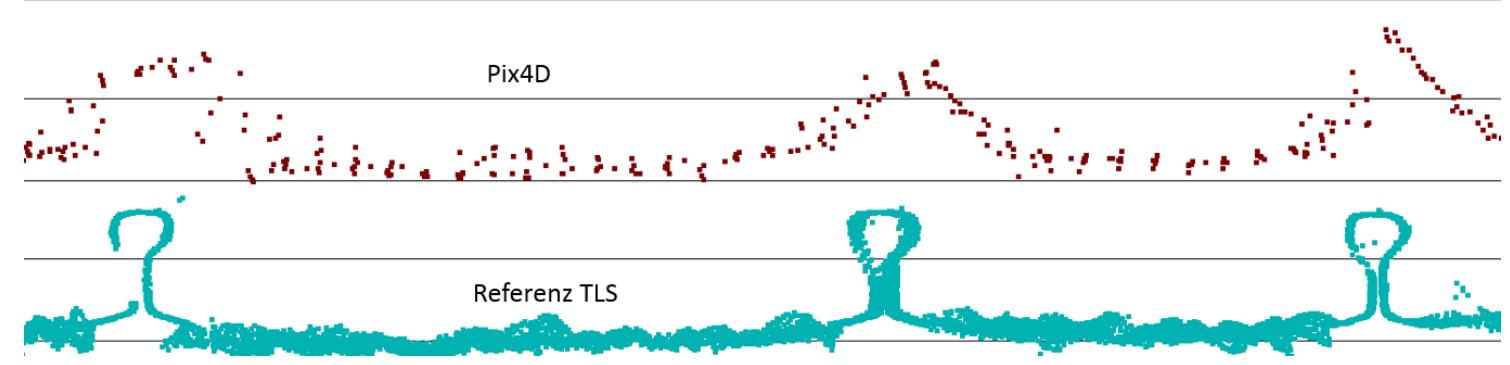

Figure 9. Profiles created with PhotoScan as shown in Figure 8, but using different filter factors compared to TLS reference 

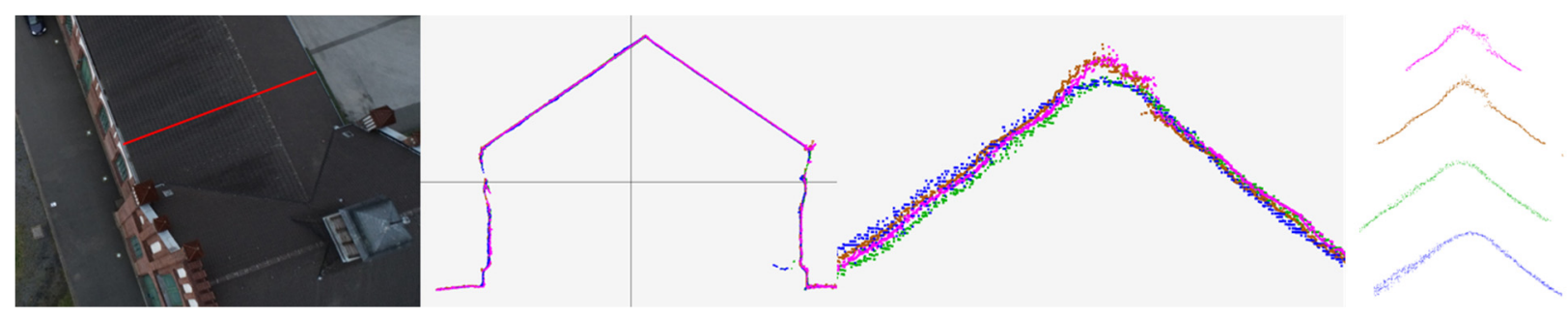

Figure 10. F.l.t.r.: Photo with profile of a building, reference point cloud from terrestrial laser scanning, dense point cloud profiles from image-based generation P4D JPG80 (pink), P4D TIF (brown), PS JPG80 (green) and PS TIF (blue)

The object "ramp" has a tarred and more or less flat surface with sharply defined edges and a significant height difference at the left edge. The heat maps in Figure 12 (top) show the differences between the dense point clouds and the TLS reference data. The range of the deviations is comparable for the data sets shown, but a systematic offset between PhotoScan and Pix4Dmapper point clouds is again visible, which confirm the results presented in section 3.1 and 3.2. Furthermore, the effects of the "aggressive" filter setting, which was applied for the generation of the dense point cloud in PhotoScan, are clearly visible at the edge of the ramp. These effects are also visible in the 3D comparison of the point clouds of the "rail tracks" object.

The results of the comparisons on the "paving stones" object are depicted in Figure 13 and 14. While in Figure 13 (left) the TLS scan data clearly shows the surface structure of the stones, this is not visible in the illustration of the image-based dense point cloud (right) of PhotoScan (TIF). The selected GSD of the aerial flight $(14 \mathrm{~mm})$ and the reduced image resolution in the matching process (quality level "medium") are obviously insufficient to adequately depict the stone structure.

The grey spots of the heat maps shown in Figure 14 result from the benches and steles set up on the site; these areas are not used in the $3 \mathrm{D}$ comparison. All in all, the results obtained so far have been confirmed without any noticeable changes depending on the available surface structure and material. The deviations of the point cloud from PhotoScan are essentially in the range of the mean average value of approx. $0 \mathrm{~mm}$, with a scatter of some millimetres both, in the positive and in the negative height
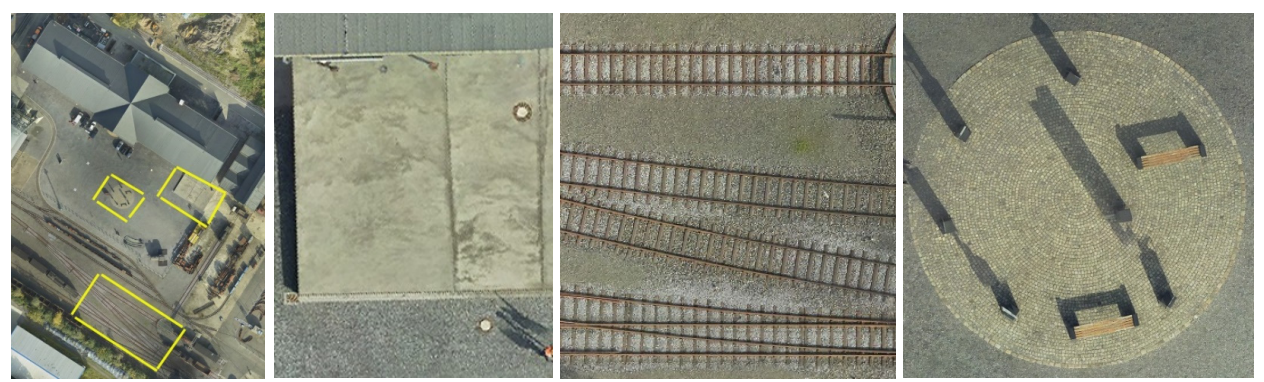

Figure 11. Overview of area-based objects (left) and detailed view (f.1.t.r.): ramp, rails and paving stones
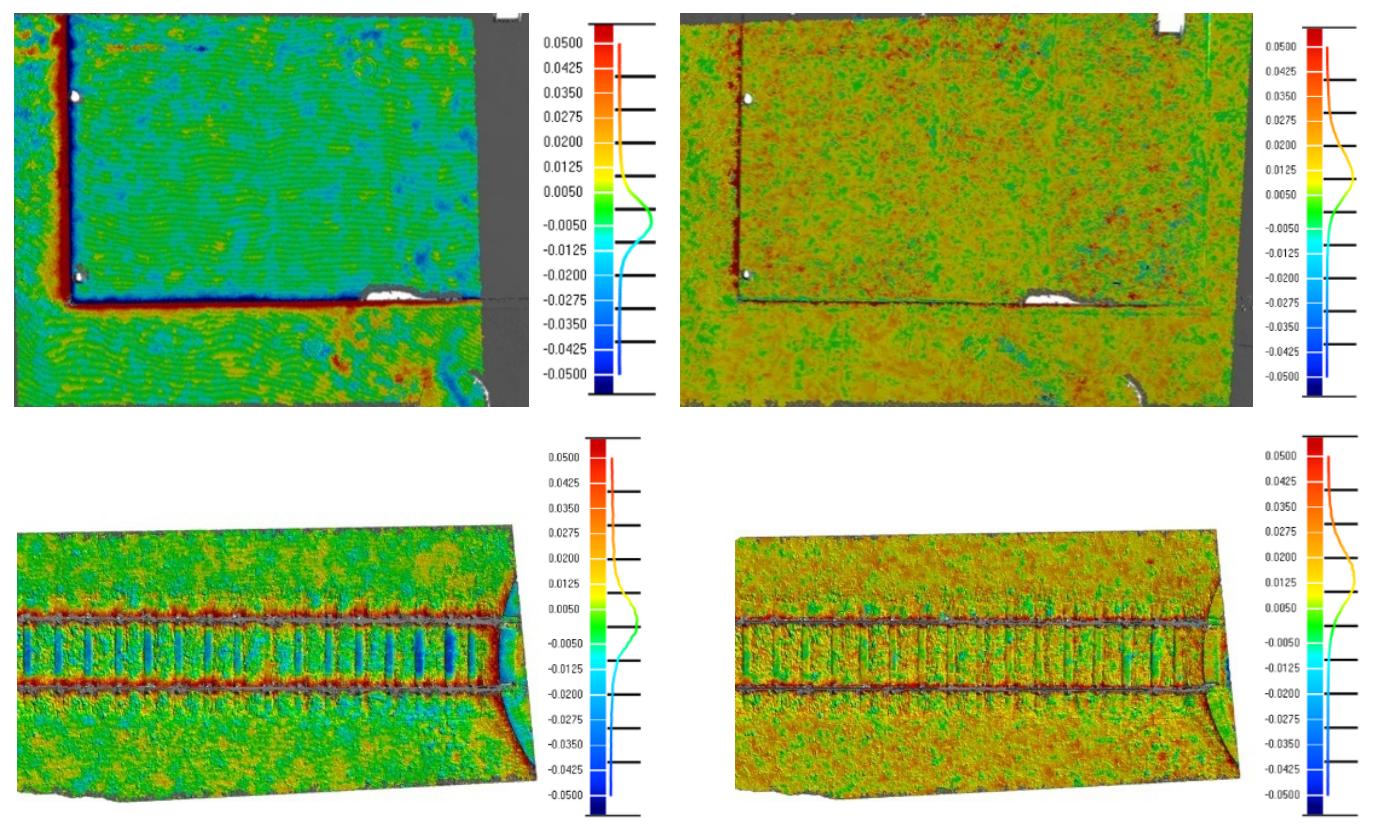

Figure 12. Vertical surface deviations of point clouds [m] compared to TLS reference data for the ramp (top) and the rails (bottom): PS TIF (left), Pix4D TIF (right) 

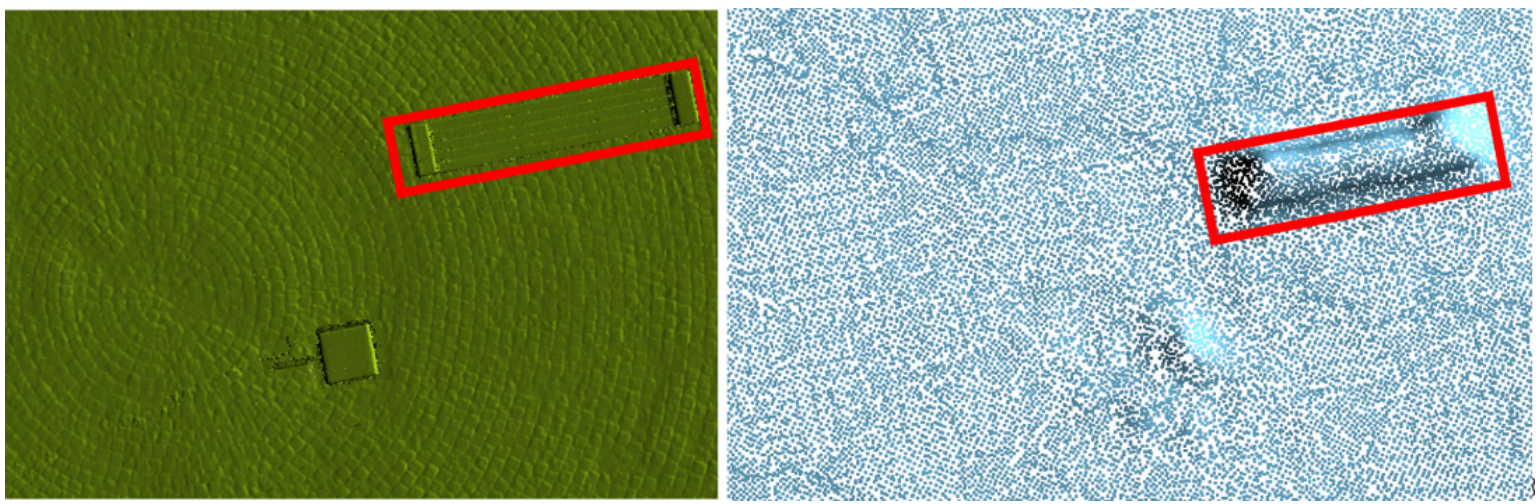

Figure 13. Reference data from terrestrial laser scanning (left), dense point cloud from PS TIF (right)
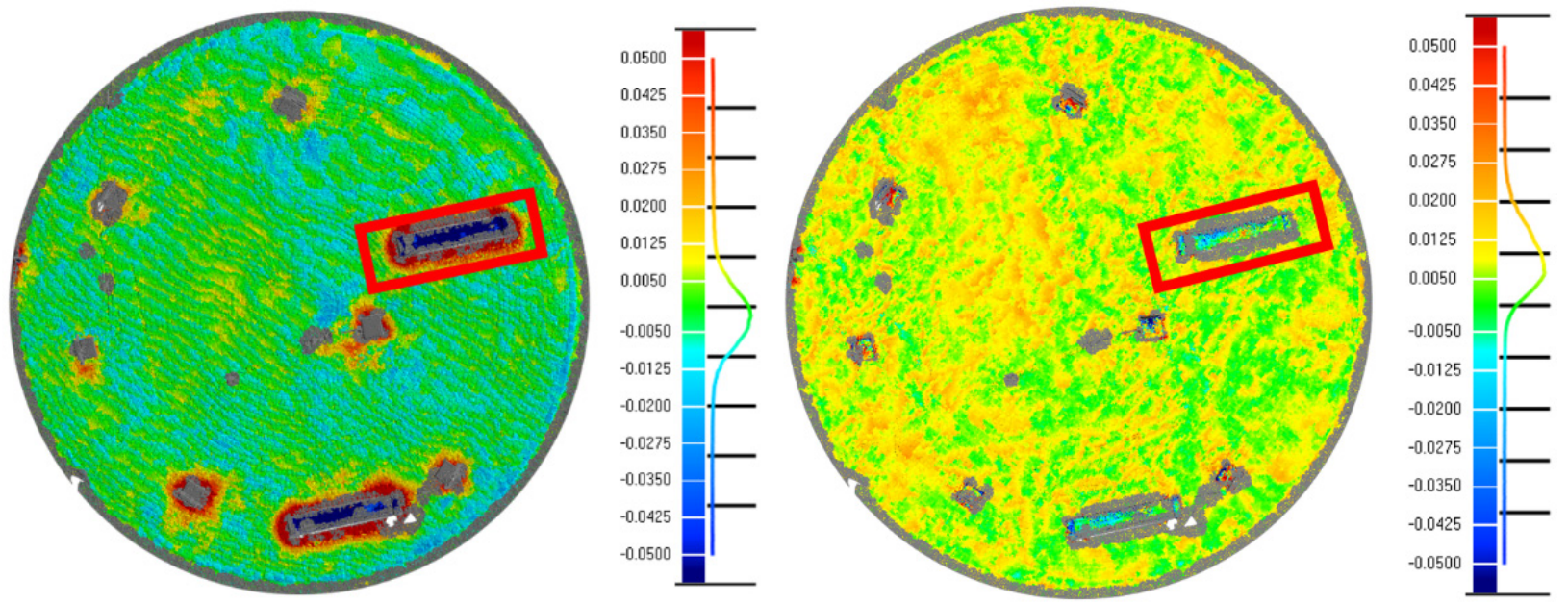

Figure 14. Colour representation of the area-based vertical deviations [m] for the paving stones: PS TIF (left) and Pix4D TIF (right). Deviations in green $\pm 5 \mathrm{~cm}$

direction. On the other side, the point cloud of Pix4Dmapper shows a clear positive shift (on average $8-10 \mathrm{~mm}$ ), which is located above the reference data.

\section{CONCLUSION AND OUTLOOK}

The quality of image-based dense point clouds is influenced by a variety of parameters. Besides the image sensor used and the quality of the image data captured, the software including the parameter settings for dense image matching and the filter algorithms are of major importance.

In the current investigation the influence of the image data format (TIF vs. JPG) in the quality of point cloud generation is negligible. The expected differences in the JPG-based point clouds due to possible compression losses compared to those from TIF data are not significant. The applied, light compression used in the Capture One software (here $80 \%$ ) clearly has only a minor effect on the geometric quality of the images. However, the effect of increased compression rates on image-based dense point cloud generation will be investigated in future tests. The pre-processed JPG images of alternative systems such as DJI (with corrected radial distortion) should also be included in these investigations, as they represent the standard image format of many UAV systems.

However, the influence of the software programs used with the corresponding standard parameters for dense point cloud generation is clearly visible in the results. On the one hand, the test data generated using Pix4Dmapper show a height offset relative to the geodetic reference of approx. $10 \mathrm{~mm}$ (using a GSD of $14 \mathrm{~mm}$ ), while on the other hand the effects of parameters for data filtering (Agisoft PhotoScan) are demonstrated, since object details are often eliminated by filtering. The effectiveness of the various filter parameters must therefore be evaluated more intensively in further tests, in order to find an optimal strategy for processing of point clouds, as already published by Mayer et al. (2018) for aerial triangulations with PhotoScan. More detailed information about all algorithms used in the software would support the analysis of the results by the user.

\section{REFERENCES}

Agisoft 2019a. Homepage Agisoft, https:/www.agisoft.com, last access 19.01.2019.

Agisoft 2019b. PhotoScan Manual - Dense Point Cloud Parameter, https://www.agisoft.com/pdf/photoscanpro_1_4_en.pdf, 22-23, last access 19.01.2019.

Coptersystems 2019. Multicopter "CS8-MM", https://coptersystems.com/produkte/cs8-mm/, last access 19.01.2019.

Haala, N., Mandlburger, G., Cramer, M., Laupheimer, D. \& Kölle, M. 2019. Kombinierte Analyse hochpräziser Punktwolken aus UAV-Photogrammetrie und -Laserscanning im Hinblick auf Setzungsmessungen. 20. Internationale Geodätische Woche Obergurgl 2019, Hanke, K. \& Weinold, T. (eds.), Herbert Wichmann Verlag, VDE Verlag GmbH, Berlin und Offenbach. 
Kersten, T. \& Lindstaedt, M. 2012. Automatic 3D Object Reconstruction from Multiple Images for Architectural, Cultural Heritage and Archaeological Applications Using Open-Source Software and Web Services. PFG - Journal of Photogrammetry, Remote Sensing and Geoinformation Science, 6, 727-740.

Kersten, T., Lindstaedt, M., Mechelke, K., Omelanowsky, D. \& Prenting, J. 2016. Low-Cost vs. High-End Systeme im Vergleich - 3D-Aufnahme der Ringwallanlage Lembecksburg auf der Nordseeinsel Föhr. Photogrammetrie, Laserscanning, Optische 3D-Messtechnik - Beiträge der Oldenburger 3D-Tage 2016, Th. Luhmann/Ch. Schumacher (eds.), Wichmann, VDE Verlag $\mathrm{GmbH}$, Berlin und Offenbach, 150-161.

Mayer, C., Gomes Pereira, L. M. \& Kersten, T. 2018. A Comprehensive Workflow to Process UAV Images for the Efficient Production of Accurate Geoinformation. CNCG2018 IX Conferência Nacional de Cartografia e Geodesia, Amadora, Portugal, Oct. 25-26.

d'Oleire-Oltmanns, S. \& Lackner, B. 2018. Evaluierung terrestrischer und UAS-basierter photogrammetrischer Datenprodukte. AGIT - Journal für Angewandte Geoinformatik, 4-2018, Wichmann Verlag, VDE Verlag GmbH Berlin Offenbach, 372-377.

Phase One 2019a. Mapping and Surveying - Focusing on the Small Details, Seeing the Large Picture. https://industrial.phaseone.com/Mapping_Surveying_Applicatio ns.aspx, last access 16.03.2019.
Phase One 2019b. Capture One. https://www.captureone.com/en/, last access 16.03.2019.

Pix4D 2019a. Homepage Pix4Dmapper. https://www.pix4d.com/product/pix4dmapper-photogrammetrysoftware, last access 19.01.2019.

Pix4D 2019b. Pix4Dmapper - Dense Point Cloud Parameter. https://support.pix4d.com/hc/en-us/articles/202557799, last access 19.01.2019.

Przybilla, H.-J., Kraft, T., Geßner, M. \& Zurhorst, A. 2017. Untersuchungen und erste Ergebnisse zur geometrischen Qualität marktgängiger Kameras für den UAV-Einsatz. Photogrammetrie, Laserscanning, Optische 3D-Messtechnik Beiträge der Oldenburger 3D-Tage 2017, Th. Luhmann/Ch. Schumacher (eds.), Wichmann - VDE Verlag, 235-246.

Przybilla, H.-J. 2019. Untersuchungen zur Leistungsfähigkeit aktueller bildgebender UAV-Sensoren. Publikationen der Deutschen Gesellschaft für Photogrammetrie, Fernerkundung und Geoinformation e.V., Band 28, Tagungsband der 39. Wissenschaftlich-Technischen Jahrestagung der DGPF, Dreiländertagung, 20.-22. Februar 2019 in Wien, 483-497.

Zhang, Z., Gerke, M., Vosselman, G. \& Yang, M. Y. 2018. Filtering Photogrammetric Point Clouds Using Standard Lidar Filters Towards DTM Generation. ISPRS Ann. Photogramm. Remote Sens. Spatial Inf. Sci., IV-2, 319-326. 